\title{
TWO NORMAL FORM THEOREMS FOR CSP PROGRAMS *
}

\author{
K.R. APT \\ Centre for Mathematics and Computer Science, Kruislaan 413, 1098 SJ Amsterdam, The Netherlands \\ Luc BOUGÉ ** \\ d'Ulm, F-75230 Paris Cédex 05, France

\section{Ph. CLERMONT} \\ ETCA, Service CTME/OP, 16 bis av. Prieur de la Côte d'Or, F-94114 Arcueil, France \\ Communicated by David Gries \\ Received 20 February 1985 \\ Revised 29 July 1985 and 13 July 1987
}

Département de Mathématiques et d'Informatique, Laboratoire d'Informatique de l'Ecole Normal Supérieure (LIENS), 45, rue

\begin{abstract}
We define two normal forms for CSP programs. In the First Normal Form, each process contains only one I/O repetitive command and all its I/O commands appear as guards of this command. In the Second Normal Form, all guards of this I/O repetitive command are $\mathrm{I} / \mathrm{O}$ guards. We describe an inductive method that transforms any CSP program into an equivalent program in first or second normal form. The notion of equivalence is discussed. It is shown that no transformation into second normal form can preserve deadlock freedom.
\end{abstract}

Keywords: CSP, normal form, partially interpreted computation, syntactic equivalence, deadlock freedom

\section{Introduction}

One of the best known theorems in Theoretical Computer Science states that every while program is equivalent to a program with one loop (see, e.g., [8]). We prove a similar result for CSP programs [9]. We exhibit two normal forms to which every CSP program can be brought. A CSP program is in a normal form (a normal program, in short) if each of its component processes contains only one $\mathrm{I} / \mathrm{O}$ repetitive command and all its $\mathrm{I} / \mathrm{O} \mathrm{com}-$ mands appear as guards in this command. There are various reasons why the study of our normal programs can be of interest.

* This work was partially supported by CNRS Project $\mathrm{C}^{3}$.

** L. Bougé is also affiliated with the Laboratoire d'Informatique, Université d'Orléans, B.P. 6759, F-45067 Orléans Cédex 02, France.
(1) Program construction-In the case of CSP programs, as opposed to while programs, several algorithms can be naturally expressed as normal programs. For example, most solutions to the distributed termination problem of Francez [5] are normal programs (see, e.g., [6,3]). For other algorithms written as normal programs, see for example [4].

(2) Verification-We found (see [1]) that a very simple proof system allows us to prove correctness of normal programs. Moreover, Queille and Sifakis [12] built a system allowing an automatic verification of finite-state normal programs. Adding to their system a preprocessor implementing the transformations described in this paper allows us to extend the use of their system to arbitrary finite-state CSP programs.

(3) Event-driven computing - In an event-driven concurrent system, local actions are triggered by 
the occurrence of external events. This type of computing is typical in the case of network protocols. It is often modeled by means of interacting automata (see, e.g., [7]). Normal programs exhibit a structure that makes this view of distributed computing more explicit as each process alternates between communications and resulting local actions.

Equivalence of concurrent programs is a delicate and difficult issue. In the last section of this paper we analyze the notion used here and indicate its limitations.

\section{Normal forms in CSP}

We assume the reader to be familiar with CSP [9]. We consider a variant of CSP without nested parallelism and where output guards are allowed. We do not consider the Distributed Termination Convention. For simplicity, we omit all declarations.

A CSP program $P$ is a parallel composition of named processes that operate on disjoint memories:

$\left[P_{1}:: S_{1}\|\ldots\| P_{k}:: S_{k}\right]$.

Each process $S$ is generated by the following grammar $(m \geqslant 1)$ :

$$
\begin{aligned}
S::= & \operatorname{skip} \mid \text { cmd }|\alpha| S_{1} ; S_{2} \mid \\
& {\left[\square_{j=1}^{m} G_{j} \rightarrow S_{j}\right] \mid *\left[\square_{j=1}^{m} G_{j} \rightarrow S_{j}\right] . }
\end{aligned}
$$

$G::=b \mid b ; \alpha$.

Here, cmd denotes an assignment, $b$ a Boolean expression, and $\alpha$ an I/O command. If a guard $G$ is of the form $b$, then it is called a purely Boolean guard, otherwise an I/O guard. Bool $(G)$ denotes the Boolean part of a guard. A guard $G$ is enabled when control is in front of it and $\operatorname{Bool}(G)$ evaluates to true. In the sequel, $b_{1} ; b_{2}$ denotes the conjunction $b_{1} \wedge b_{2}$ of Boolean expressions.

2.1. Definition. A process $S$ is in first normal form if it is of the form $S_{0}$ or

$S_{0} ; *\left[\square_{j=1}^{m} G_{j} \rightarrow S_{j}\right]$, where $m \geqslant 1$ and none of the $S_{j}$ contains an $\mathrm{I} / \mathrm{O}$ command.

2.2. Definition. A process $S$ is in second normal form if it is of the form $S_{0}$ or

$S_{0} ; *\left[\square_{j=1}^{m} G_{j} \rightarrow S_{j}\right]$

where $m \geqslant 1$, none of the $S_{j}$ contains any $\mathrm{I} / \mathrm{O}$ command and, moreover, all of the $G_{j}$ are I/O guards.

A CSP program is in first (respectively second) normal form if all its component processes are.

\section{The transformations}

\subsection{First normal form}

We now describe a function $N F_{1}$ that transforms each process $S$ into a process $S^{\prime}=N F_{1}(S)$ in first normal form. We proceed by induction on the structure of $S$. We assume an infinite set $Z$ of fresh Boolean variables $z_{1}, z_{2}, \ldots$ We omit indices when no confusion can arise.

Base case. When $S$ is skip or an atomic assignment command, $S$ is already in first normal form, so $N F_{1}(S)=S$.

$I / O$ command. Suppose $S$ is an I/O command $\alpha$. Select a fresh variable $z$ from $Z$ and define $N F_{1}(S)=z:=$ true $; *[z ; \alpha \rightarrow z:=$ false $]$.

Sequential composition. Suppose $S$ is of the form $S_{1} ; S_{2}$. By induction, we have

$N F_{1}\left(S_{i}\right)=$ Init $_{i} ; *\left[\square_{j=1}^{m_{i}} G_{j}^{i} \rightarrow S_{j}^{i}\right]$

where we can assume that sets $Z_{1}$ and $Z_{2}$ of fresh variables for each $S_{i}$ are disjoint. Let $z_{1}$ and $z_{2}$ be two variables of $Z \backslash Z_{1} \cup Z_{2}$. Define $N F_{1}(S)$ as follows:

$$
\begin{aligned}
& \text { Init }_{1} ; z_{1}:=\text { true; } z_{2}:=\text { false; } \\
& *\left[\square_{j=1}^{m_{1}} z_{1} ; G_{j}^{1} \rightarrow S_{j}^{1} ;\right. \text { TEST } \\
& \left.\quad \square_{j=1}^{m_{2}} z_{2} ; G_{j}^{2} \rightarrow S_{j}^{2}\right]
\end{aligned}
$$


where TEST stands for

$$
\begin{aligned}
& {\left[\square_{j=1}^{m_{1}} \operatorname{Bool}\left(G_{j}^{1}\right) \rightarrow\right. \text { skip }} \\
& \square \bigwedge_{j=1}^{m_{1}} \neg \operatorname{Bool}\left(G_{j}^{1}\right) \rightarrow z:=\text { false; } \\
& \left.z_{2}:=\text { true } ; \text { Init }_{2}\right] .
\end{aligned}
$$

Intuitively, $z_{1}$ is true when control is still in $S_{1}$ and $z_{2}$ is true when control is in $S_{2}$.

Repetitive command. Suppose $S$ is of the form

$$
\text { *[ } \left.\square_{i=1}^{m} H_{i} \rightarrow R_{i}\right] \text {. }
$$

By induction, we have

$$
N F_{1}\left(R_{i}\right)=\text { Init }_{i} ; *\left[\square_{j=1}^{m_{i}} G_{j}^{i} \rightarrow S_{j}^{i}\right]
$$

where we can assume without loss of generality that sets $Z_{i}$ of fresh variables for each $R_{i}$ are pairwise disjoint. Let turn $_{i}, i=1,2, \ldots, m$ be fresh variables of $Z \backslash \cup_{i=1}^{m} Z_{i}$. Define $N F_{1}(S)$ as follows:

$$
\begin{aligned}
& \text { turn }_{1}:=\text { false } ; \ldots ; \text { turn }_{m}:=\text { false } \\
& *\left[\square_{i=1}^{m} \bigwedge_{i=1}^{m} \neg_{\text {turn }_{i}}^{m} ; H_{i} \rightarrow \text { turn }_{i}:=\text { true } ;\right.
\end{aligned}
$$

$$
\text { Init }_{i} ; \text { TEST }_{i}
$$

$$
\left.\square_{\substack{i=1,2, \ldots, m_{j} \\ j=1,2, \ldots, m_{i}}} \operatorname{turn}_{i} ; G_{j}^{i} \rightarrow S_{j}^{i} ; \text { TEST }_{i}\right]
$$

where $T E S T_{i}$ stands for

$$
\begin{aligned}
& {\left[\square_{k=1}^{m_{i}} \operatorname{Bool}\left(G_{k}^{i}\right) \rightarrow\right. \text { skip }} \\
& \left.\square \bigwedge_{k=1}^{m_{i}} \neg \operatorname{Bool}\left(G_{k}^{i}\right) \rightarrow \text { turn }_{i}:=\text { false }\right] .
\end{aligned}
$$

Intuitively, turn ${ }_{i}$ holds when control is in subpro- gram $R_{i}$. Then, TEST $T_{i}$ indicates whether $R_{i}$ is terminated and turn $_{i}$ is reset to false if this is the case.

Alternative command. Suppose $S$ is of the form

$\left[\square_{i=1}^{m} H_{i} \rightarrow R_{i}\right]$.

By induction, we have

$N F_{1}\left(R_{i}\right)=$ Init $_{i} ; *\left[\square_{j=1}^{m_{i}} G_{j}^{i} \rightarrow S_{j}^{i}\right]$

in first normal form. Using a new variable $z$ from $Z \backslash \bigcup_{i=1}^{m} Z_{i}$, we first transform $S$ into the following process $S^{\prime}$ :

$$
z:=\text { true; }
$$

$$
\begin{aligned}
& {\left[\square_{i=1}^{m} \operatorname{Bool}\left(H_{i}\right) \rightarrow \text { skip }\right] ;} \\
& *\left[\square_{i=1}^{m} z ; H_{i} \rightarrow R_{i} ; z:=\text { false }\right] .
\end{aligned}
$$

Those two processes are related as follows. First suppose that $S$ fails. This occurs when all conditions $\operatorname{Bool}\left(H_{i}\right)$ evaluate to false initially. Then, $S^{\prime}$ fails much in the same way. Suppose $S$ does not fail; then at least one of those conditions evaluates to true. In $S^{\prime}$, the alternative command then boils down to skip. In the repetitive command, the conditions are evaluated again and yield the same results as before, because processes operate on disjoint memories. At least one of them is thus guaranteed to evaluate to true, $S^{\prime}$ does not fail either, and behaves subsequently like $S . N F_{1}(S)$ is the result of applying transformation $N F_{1}$ to process $S^{\prime}$.

This concludes the presentation of the transformation $N F_{1}$.

Property A. For each process $S, N F_{1}(S)$ is a process in first normal form. The only atomic commands in $N F_{1}(S)$ in which variables from $Z$ appear are of the form $z:=$ true or $z:=$ false. 


\subsection{Second normal form}

We now describe a procedure $N F_{2}$ that transforms each process $S$ in first normal form into a process $N F_{2}(S)$ in second normal form. A process $S$ in first normal form all of whose external guards contain an $\mathrm{I} / \mathrm{O}$ command (or that contains no $\mathrm{I} / \mathrm{O}$ command) is already in second normal form and we put $N F_{2}(S)=S$. Otherwise, it can be written as

Init;

$$
\begin{array}{r}
{\left[\square_{i=1}^{m} G_{i} \rightarrow S_{i}\right.} \\
\left.\square_{j=1}^{n} H_{j} \rightarrow T_{j}\right]
\end{array}
$$

with $m>0$ and $n>0$, where all guards $G_{i}$ are purely Boolean and all guards $H_{j}$ do contain an $\mathrm{I} / \mathrm{O}$ command. Let CHOOSE be the following command:

$$
\begin{aligned}
& \text { turn }_{1}:=\text { false } ; \ldots ; \text { turn }_{n}:=\text { false; } \\
& *\left[\square_{i=1}^{m} \bigwedge_{k=1}^{n} \neg \text { turn }_{k} ; G_{i} \rightarrow S_{i}\right. \\
& \left.\square_{i=1}^{n} \bigwedge_{k=1}^{n} \neg \text { turn }_{k} ; \operatorname{Bool}\left(H_{j}\right) \rightarrow \text { turn }_{j}:=\text { true }\right] .
\end{aligned}
$$

Execution of CHOOSE consists of some iterations of the repetitive command

* $\left[\square_{i=1}^{m} G_{i} \rightarrow S_{i}\right]$

which contains no I/O command followed by the selection of an I/O guard $H_{j}$, provided its Boolean part $\operatorname{Bool}\left(H_{j}\right)$ evaluates to true. We then define $\mathrm{NF}_{2}(\mathrm{~S})$ to be the following process:

Init ; CHOOSE;

* $\left[\square_{j=1}^{n}\right.$ turn $_{j} ; H_{j} \rightarrow T_{j} ;$ CHOOSE $]$.

Observe that $\operatorname{Bool}\left(H_{j}\right)$ is evaluated twice, once within CHOOSE and then again within $H_{j}$. Both evaluations return necessarily the same result because processes operate on disjoint memories.

Property B. For each process $S$ in first normal form, $N F_{2}(S)$ is in second normal form. The only atomic commands in $N F_{2}(S)$ in which variables from $Z$ appear are of the form $z:=$ true and $z:=$ false.

\subsection{Homogeneous processes}

For certain processes, it is possible to describe a direct transformation that yields a process in second normal form. A process is homogeneous if, in each repetitive or alternative command, either all guards are purely Boolean or all guards contain an $\mathrm{I} / \mathrm{O}$ command. Observe that a homogeneous process is in first normal form if and only if it is in second normal form. If we can modify procedure $N F_{1}$ so as to preserve homogeneity, then it will transform homogeneous processes into processes in second normal form. The only part of $N F_{1}$ that does not preserve homogeneity is the one dealing with a repetitive command $S$

* $\left[\square_{i=1}^{m} H_{i} \rightarrow R_{i}\right]$

whose guards are all purely Boolean. In this case, let $S W I T C H$; be

$$
\begin{aligned}
& {\left[\square_{i=1}^{m} H_{i} \rightarrow \text { turn }_{i}:=\text { true; } \text { Init }_{i}\right.} \\
& \left.\square \bigwedge_{i=1}^{m} \neg H_{i} \rightarrow \text { skip }\right] .
\end{aligned}
$$

Then, assuming the notation used in $N F_{1}(S)$, the transformed process is

turn $_{1}:=$ false $;. . ;$ turn $_{m}:=$ false;

SWITCH;

$$
\begin{aligned}
* \prod_{\substack{i=1,2, \ldots, m \\
j=1,2, \ldots, m_{i}}}^{\square} \text { turn }_{i} ; G_{j}^{i} \rightarrow S_{j}^{i} ; \text { TEST }_{i} ; \\
{\left.\left[\text { turn }_{i} \rightarrow \operatorname{skip} \square \neg \text { turn }_{i} \rightarrow \text { SWITCH }\right]\right] . }
\end{aligned}
$$


Denote this modified transformation by $N F_{1}^{\prime}$. Here, variables turn $_{i}$ are used for the same purpose as before. Setting a variable turn $_{i}$ to true can take place in the SWITCH command only.

Property C. For each homogeneous process $S$, $N F_{1}^{\prime}(S)$ is in second normal form. The only atomic commands in $N F_{1}^{\prime}$ in which variables from $Z$ appear are of the form $z:=$ true and $z:=$ false.

\section{A notion of equivalence}

We now wish to make precise in what sense every process $S$ is equivalent to the process $S^{\prime}$ generated in Section 3 by the transformations $N F_{1}, N F_{2}$, and $N F_{1}^{\prime}$. To this purpose, we first associate with each process $S$ a regular language $L(S)$. Intuitively, $L(S)$ is the set of all uninterpreted possible computations of process $S$ according to Plotkin's semantics [11].

The language $L(S)$ is over the alphabet consisting of atomic actions $\mathrm{cmd}, \mathrm{I} / \mathrm{O}$ commands $\alpha$, Boolean conditions $b$, plus two special tokens $\langle s k i p\rangle$ and $\langle$ fail $\rangle$ that denote respectively termination and failure. $L(S)$ is defined inductively as follows.

$$
\begin{aligned}
& L(\text { skip })=\{\langle\text { skip }\rangle\}, \\
& L(\text { cmd })=\{\langle\text { cmd }\rangle\}, \\
& L(\alpha)=\{\langle\alpha\rangle\}, \\
& L(G)= \begin{cases}\{\langle b\rangle\langle\alpha\rangle\} & \text { if } G=b ; \alpha, \\
\{\langle b\rangle\langle\text { skip }\rangle\} & \text { if } G=b,\end{cases} \\
& L\left(S_{1} ; S_{2}\right)=L\left(S_{1}\right) \cdot L\left(S_{2}\right), \\
& L\left(\left[G_{1} \rightarrow S_{1} \square \cdots \square G_{m} \rightarrow S_{m}\right]\right) \\
&=\left[\left(L\left(G_{1}\right) \cdot L\left(S_{1}\right)\right) \cup \cdots \cup\left(L\left(G_{m}\right) \cdot L\left(S_{m}\right)\right)\right] \\
& \quad \cdot\{\langle\text { Bool }\rangle\langle\text { fail }\rangle\}, \\
& L\left(*\left[G_{1} \rightarrow S_{1} \square \cdots \square G_{m} \rightarrow S_{m}\right]\right) \\
&=\left[\left(L\left(G_{1}\right) \cdot L\left(S_{1}\right)\right) \cup \cdots \cup\left(L\left(G_{m}\right) \cdot L\left(S_{m}\right)\right)\right]^{*} \\
& \cdot\{\langle\text { Bool }\rangle\langle\text { skip }\rangle\},
\end{aligned}
$$

where Bool stands for

$\neg \operatorname{Bool}\left(G_{1}\right) \wedge \cdots \wedge \neg \operatorname{Bool}\left(G_{m}\right)$.
Observe how the appropriate exit conditions are reflected.

To obtain the desired equivalence, we partially interpret the computations by evaluating the commands and conditions associated with auxiliary variables. In the processes generated by the transformations of Section 3, they can be of the following type exclusively:

$$
\begin{aligned}
& z:=\text { true }, \\
& z:=\text { false, } \\
& B\left(z_{1}, \ldots, z_{m}, b_{1}, \ldots, b_{n}\right),
\end{aligned}
$$

where $B$ is some Boolean combination of its arguments. When evaluating the condition, variables $z_{i}$ are substituted with their current value, true or false. The condition is said to be unsatisfiable if the resulting formula is equivalent to false as a formula of the predicate calculus with variables $b_{j}$. The condition is satisfiable if it is not unsatisfiable. Then we exclude contradictory computations, i.e., those that violate the rule that the selected Boolean conditions are all satisfiable. Finally, we erase all skip's and assignments $z:=$ true and $z:=$ false to auxiliary variables. Also, we merge adjacent Boolean formulas into their Boolean conjunction and reduce the resulting formula to some normal form (say, a conjunction of disjunctions for definiteness). Tautologies are then erased. Let $L^{\prime}(S)$ be the resulting language. It is a language over the alphabet consisting of atomic actions cmd, I/O commands $\alpha$, Boolean formula $B\left(b_{1}, \ldots, b_{n}\right)$, and token $\langle$ fail $\rangle$. We say that two processes $S_{1}$ and $S_{2}$ are equivalent with respect to a set $Z$ of auxiliary variables if

$L^{\prime}\left(S_{1}\right)=L^{\prime}\left(S_{2}\right)$.

This equivalence can be best understood with an example. Consider the processes

$S_{1}=\alpha$

and

$S_{2}=z:=$ true $; *[z ; \alpha \rightarrow z:=$ false $]$.

Then

$L\left(S_{1}\right)=L^{\prime}\left(S_{1}\right)=\{\langle\alpha\rangle\}$. 
On the other hand,

$$
\begin{aligned}
L\left(S_{2}\right)= & \langle z:=\text { true }\rangle(\langle z\rangle\langle\alpha\rangle\langle z:=\text { false }\rangle)^{*} \\
& \langle\neg z\rangle\langle s k i p\rangle .
\end{aligned}
$$

After the interpretation of actions related to the variable $z$ we obtain the following set of words:

$$
\begin{aligned}
& \{\langle z:=\text { true }\rangle\langle\text { true }\rangle\langle\alpha\rangle\langle z:=\text { false }\rangle\langle\neg \text { false }\rangle\langle\text { skip }\rangle, \\
& \langle z:=\text { true }\rangle\langle\text { true }\rangle\langle\alpha\rangle\langle z:=\text { false }\rangle \\
& \left.(\langle\text { false }\rangle\langle\alpha\rangle\langle z:=\text { false }\rangle)^{*}\langle\neg \text { false }\rangle\langle\text { skip }\rangle\right\} .
\end{aligned}
$$

Here, only the computation

$$
\langle z:=\text { true }\rangle\langle\text { true }\rangle\langle\alpha\rangle\langle z:=\text { false }\rangle\langle\neg \text { false }\rangle\langle\text { skip }\rangle
$$

is not contradictory. Deleting from it all assignments to the variables of $Z$ and skip's, reducing sequences of adjacent Boolean formulas to their normal form and erasing tautologies, we get $\langle\alpha\rangle$ as desired.

We have the following theorems, whose tedious but straightforward proofs are omitted.

4.1. Theorem. Both $N F_{1}(S)$ and $N F_{1}^{\prime}(S)$ are equivalent to $S$ with respect to set $Z$ of auxiliary variables.

4.2. Theorem. $N F_{2}(S)$ is equivalent to $S$ with respect to set $Z$ of auxiliary variables.

These equivalences are on the level of processes considered in isolation. The following theorem states some of its semantic consequences. By a state we mean a function assigning values to each of the variables. We consider $\perp$ as a special state indicating divergence. Given a CSP program $P$, we define its meaning $\mathscr{M}[11]$ by

$$
\mathscr{M}[11](\sigma)
$$

$=\{\tau, \tau$ is the final state of a properly terminating computation starting in state $\sigma\}$ $\cup\{\perp$, there exists a diverging computation of $P$ starting in state $\sigma$ \}.

For two sets $\Sigma_{1}$ and $\Sigma_{2}$, and a set of variables $Z$ we define

$\Sigma_{1}=\Sigma_{2} \bmod Z$

$$
\text { iff }\left\{\sigma \backslash Z, \sigma \in \Sigma_{1}\right\}=\left\{\sigma \backslash Z, \sigma \in \Sigma_{2}\right\}
$$

where $\sigma \backslash Z$ is the restriction of $\sigma$ to the variables not in $Z$. We now say that two programs CSP $P_{1}$ and $P_{2}$ are equivalent modulo $Z$ if, for all states $\sigma$, $\mathscr{M}\left[P_{1}\right](\sigma)=\mathscr{M}\left[P_{2}\right](\sigma) \bmod Z$.

Note that this equivalence definition does not take into account possible deadlocks. We can finally state the appropriate theorem.

4.3. Theorem. Let $S_{1}$ and $S_{2}$ be two equivalent processes with respect to a set $Z$ of auxiliary variables. Let

$\mathscr{C}=\left[Q_{1}:: T_{1}\|\cdots\| Q_{k}::[]\|\cdots\| Q_{n}:: T_{n}\right]$

be a context, and let $P_{i}=\mathscr{C}\left[S_{i}\right], i=1,2$, be the CSP programs obtained by plugging process $S_{i}$ into the context $\mathscr{C}$. Then, $P_{1}$ and $P_{2}$ are equivalent modulo $Z$.

Thus, up to deadlock, $P_{1}$ and $P_{2}$ exhibit the same functional behaviors.

\section{Discussion}

The equivalence relation introduced in Section 4 seems at first sight very strong, since it is basically a syntactic equivalence. However, it is concerned only with some form of traces (in the sense of [10]) of computations. Semantically, it assures only Theorem 4.3. In particular, it does not capture all relevant semantic properties naturally associated with concurrent programs, like deadlock freedom.

Indeed, consider two processes $S$ and $S^{\prime}$ where

$$
\begin{gathered}
S=[\text { true } ; Q ? x \rightarrow \text { skip } \\
\square \text { true } ; Q ! x \rightarrow \text { skip }], \\
S^{\prime}=[\text { true } \rightarrow Q ? x ; \text { skip } \\
\square \text { true } \rightarrow Q ! x ; \text { skip }] .
\end{gathered}
$$

Then, $S$ and $S^{\prime}$ are equivalent in the sense of Section 4. However, the program

$[P:: S \| Q:: P ? y]$

cannot deadlock whereas the program

$\left[P:: S^{\prime} \| Q:: P ? y\right]$ 
can. Thus, plugging equivalent processes in the same context, here

$[P::[] \| Q:: P ? y]$,

can yield two programs that behave differently. We can prove that Theorem 4.2 cannot be strengthened so that deadlock freedom is preserved in the above sense. This follows from the following theorem.

5.1. Theorem. Let $[P:: S \| Q:: T]$ be a program in second normal form. Suppose that it admits two properly terminating computations, $C_{1}$ with some communication and $C_{2}$ without any communication. Then, it admits a deadlocked computation.

Proof. Construct the deadlocked computation as follows. First take all steps carried out by $P$ in $C_{1}$ until the I/O command selected for its first communication is reached. Then, append to it all steps carried out by $Q$ in $C_{2}$. In the resulting computation, $Q$ properly terminates whereas $P$ reaches an entry to a repetitive command with all guards containing an I/O guard. Thus, a deadlock arises (observe that this would not necessarily hold if the Distributed Termination Convention of CSP were used).

This shows that first normal form cannot be reduced to second normal form when deadlock freedom is to be preserved. This can be interpreted as a statement that use of nonhomogeneous guards strictly increases the expressive power of CSP.

\section{Note}

The first version of this paper appeared as a report [2]. After having written the present ver- sion, we learned of a related work by Zöbel [13]. Zöbel proposes transformations similar to ours, but does not elaborate on the underlying notion of equivalence.

\section{References}

[1] K.R. Apt, Correctness proofs of distributed termination algorithms, ACM Trans. Programm. Languages \& Systems 8 (3) (1986) 388-405.

[2] K.R. Apt and Ph. Clermont, Two Normal Form Theorems for CSP Programs, Rept. No. RC 10975, IBM T.J. Watson Research Center, Yorktown Heights, NY, 1985.

[3] K.R. Apt and J.-L. Richier, Real time clocks versus virtual clocks, in: Proc. Internat. Summer School on Control Flow and Data Flow: Concepts of Distributed Programming, NATO ASI Series F14 (Springer, Berlin, 1985).

[4] L. Bougé, Genericity and Symmetry for Distributed Systems: The Case of CSP, Thèse d'État, Univ. Paris 7, 1987; Rept. No. 87/2, LIENS, Paris, 1987 (in French).

[5] N. Francez, Distributed termination, ACM Trans. Programm. Languages \& Systems 2 (1) (1980) 42-55.

[6] N. Francez, M. Rodeh and M. Sintzoff, Distributed termination with interval assertion, Proc. Internat. Coll. on Formalization of Programming Concepts, Peniscola, Spain, Lecture Notes in Computer Science, Vol. 107 (Springer, Berlin, 1981).

[7] M.G. Gouda, Closed covers: To verify progress for communicating finite state machines, IEEE Trans. Software Engrg. SE-10 (6) (1984) 846-855.

[8] D. Harel, On folk theorems, Comm. ACM 23 (7) (1980) 379-389.

[9] C.A.R. Hoare, Communicating sequential processes, Comm. ACM 21 (8) (1978) 666-677.

[10] C.A.R. Hoare, Some properties of predicate transformers, J. ACM 25 (3) (1978) 461-480.

[11] G. Plotkin, An operational semantics for CSP, in: D. Bjørner, ed., Formal Description of Programming Concepts, IFIP TC-2 Working Conf., Garmish-Partenkirchen, Fed. Rep. Germany, 1982 (North-Holland, Amsterdam, 1983) 199-223.

[12] J.-P. Queille and J. Sifakis, Specification and verification of concurrent systems in CESAR, in: Proc. 5th Internat. Symp. on Programming, Paris, 1981.

[13] D. Zöbel, Normal Form Transformations for Programs in CSP, Rept., EWH Rhld.-Pf., Abteilung Koblenz, Seminar für Informatik, 1987. 